\title{
Coupling of surface plasmons between two silver films in a $\mathrm{Ag} / \mathrm{SiO} / \mathrm{Ag}$ plasmonic thermal emitter with grating structure
}

\author{
Yi-Han Ye, ${ }^{\text {a) }}$ Yu-Wei Jiang, Ming-Wei Tsai, Yi-Tsung Chang, Chia-Yi Chen, \\ Dah-Ching Tzuang, Yi-Ting Wu, and Si-Chen Lee ${ }^{\text {b) }}$ \\ Department of Electrical Engineering, Graduate Institute of Electronics Engineering, \\ National Taiwan University, Taipei 10617, Taiwan
}

(Received 13 May 2008; accepted 8 December 2008; published online 29 December 2008)

\begin{abstract}
The reflection and emission spectra of $\mathrm{Ag} / \mathrm{SiO}_{2} / \mathrm{Ag}$ trilayer plasmonic thermal emitters with different $\mathrm{SiO}_{2}$ thicknesses are investigated. The top $\mathrm{Ag}$ film is perforated with periodic slits. It is found that the coupling of surface plasmons at the top and bottom $\mathrm{Ag} / \mathrm{SiO}_{2}$ interface results in the redshift in thermal emission peaks. In this $\mathrm{Ag} / \mathrm{SiO}_{2} / \mathrm{Ag}$ plasmonic thermal emitter, the electromagnetic field exhibits either Fabry-Pérot resonance or propagating surface plasmons depending on the thickness of $\mathrm{SiO}_{2}$ layer. By varying the thickness of $\mathrm{SiO}_{2}$ layer, transition from localized to grating-coupled propagating surface plasmon modes is observed. (C) 2008 American Institute of Physics. [DOI: 10.1063/1.3058767]
\end{abstract}

Surface plasmons (SPs) are electromagnetic excitations that occur at the metal/dielectric interface and result from the interaction between light and free electrons of metal. ${ }^{1}$ One of the applications of SPs is in light emitting device, which has been demonstrated by using one-dimensional gratings $^{2-5}$ and two-dimensional periodic metal hole array. ${ }^{6-9}$ Theoretical studies based on plasmonic thermal emitter have also been reported. ${ }^{10,11}$ For plasmonic thermal emitter with one-dimensional metallic grating, nonpropagating localized surface plasmon polariton (LSPP) mode, which resulted from the coupling of SPs at the top and bottom metal/ dielectric interface are observed. ${ }^{3,12}$ In this letter, the reflection and thermal radiation spectra of the narrow-bandwidth $\mathrm{Ag} / \mathrm{SiO}_{2} / \mathrm{Ag}$ trilayer plasmonic thermal emitters with top metallic grating structure are studied. A transition between LSPP and propagating SP modes is experimentally shown by varying the thickness of $\mathrm{SiO}_{2}$ layer. Moreover, the coupling effect of top and bottom SPs at the $\mathrm{Ag} / \mathrm{SiO}_{2}$ interface is revealed.

The fabrication processes of the $\mathrm{Ag} / \mathrm{SiO}_{2} / \mathrm{Ag}$ trilayer plasmonic thermal emitter are described as follows: a $300 \mathrm{~nm}$ Mo layer was deposited by sputtering on the back of the double-polished $n$-type Si substrate as a heating source. $20 \mathrm{~nm}$ Ti used as an adhesive layer followed by $200 \mathrm{~nm} \mathrm{Ag}$ film were deposited on the front side of the Si substrate by electron beam evaporation. Then $\mathrm{SiO}_{2}$ layers with different thicknesses were deposited on top of Ag layer by plasma enhanced chemical vapor deposition. After photolithography, a negative photoresist layer was patterned with a gratingshape array. Then a $100 \mathrm{~nm} \mathrm{Ag}$ film was deposited onto the patterned photoresist layer and lifted off to complete the processes. The lattice constant $a$ of the grating is $3 \mu \mathrm{m}$ and the metal line width $d$ is $1.6 \mu \mathrm{m}$. Figures 1(a) and 1(b) show the side and top views of device structure, respectively. The radiation area of the device is $1 \mathrm{~cm}^{2}$. By sending adequate $\mathrm{dc}$ current through the back Mo metal contact, the plasmonic thermal emitter was heated up and radiated infrared in vacuum chamber. A PerkinElmer 2000 Fourier transform in-

\footnotetext{
${ }^{a)}$ Electronic mail: r95943049@ntu.edu.tw.

${ }^{b)}$ Electronic mail: sclee@cc.ee.ntu.edu.tw.
}

frared spectrometer was adopted to measure the radiation spectra of thermal emitters at $260{ }^{\circ} \mathrm{C}$. The reflection spectra were measured by a Bruker IFS $66 v / s$ with a spectral resolution of $8 \mathrm{~cm}^{-1}$. The incident angles $\theta$ of light to the normal of the metal surface were scanned from $12^{\circ}$ to $65^{\circ}$ in $1^{\circ}$ step in the $x z$-plane as shown in Fig. 1(a).

Figures 2(a)-2(d) show the dispersion relations of SPs in plasmonic thermal emitters with different $\mathrm{SiO}_{2}$ layer thicknesses $t(t=0,20,60$, and $170 \mathrm{~nm})$. The dispersion relations of SPs along the $x$ axis (dark lines) are extracted from the minima of the reflection spectra at various incident angles (from $12^{\circ}$ to $65^{\circ}$ ). The grayscale from dark to bright represents the reflectivity from low to high. For an ideal planar silver layer, the reflectivity should be near unity in the midinfrared spectral range, therefore no reflection dips exist. When silver film is perforated with periodic slits, incident light, which satisfies that the momentum conservation law for SPs can couple with SPs at the metal/dielectric interface. Therefore less light is reflected, which causes the reflection dips in measurement. The momentum conservation law for SPs in grating structure is given by $k_{\mathrm{sp}}=k_{x}+i G$, where $k_{\mathrm{sp}}$ is the SP wave vector, $k_{x}=(2 \pi / \lambda) \sin \theta$ is the in-plane wave vector, $G=2 \pi / a$ denotes the reciprocal lattice vector, and $i$ is an integer. In Fig. 2(a), two grating-coupled SP modes, Ag/ air $[+1]$ and $\mathrm{Ag} /$ air $[-1]$, intersect with the $y$ axis at about

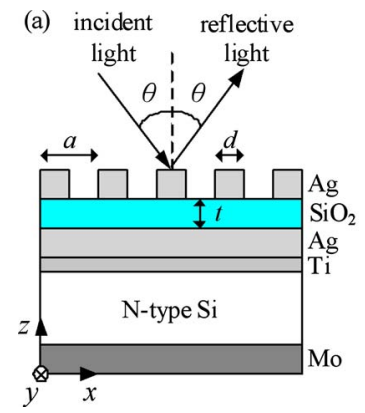

(b)

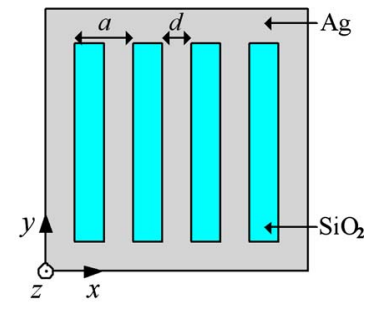

FIG. 1. (Color online) The schematic diagrams showing the (a) side and (b) top views of plasmonic thermal emitter. The top Ag metal is perforated with grating array with a lattice constant $a$ of $3 \mu \mathrm{m}$ and $\mathrm{Ag}$ line width $d$ of $1.6 \mu \mathrm{m}$. 

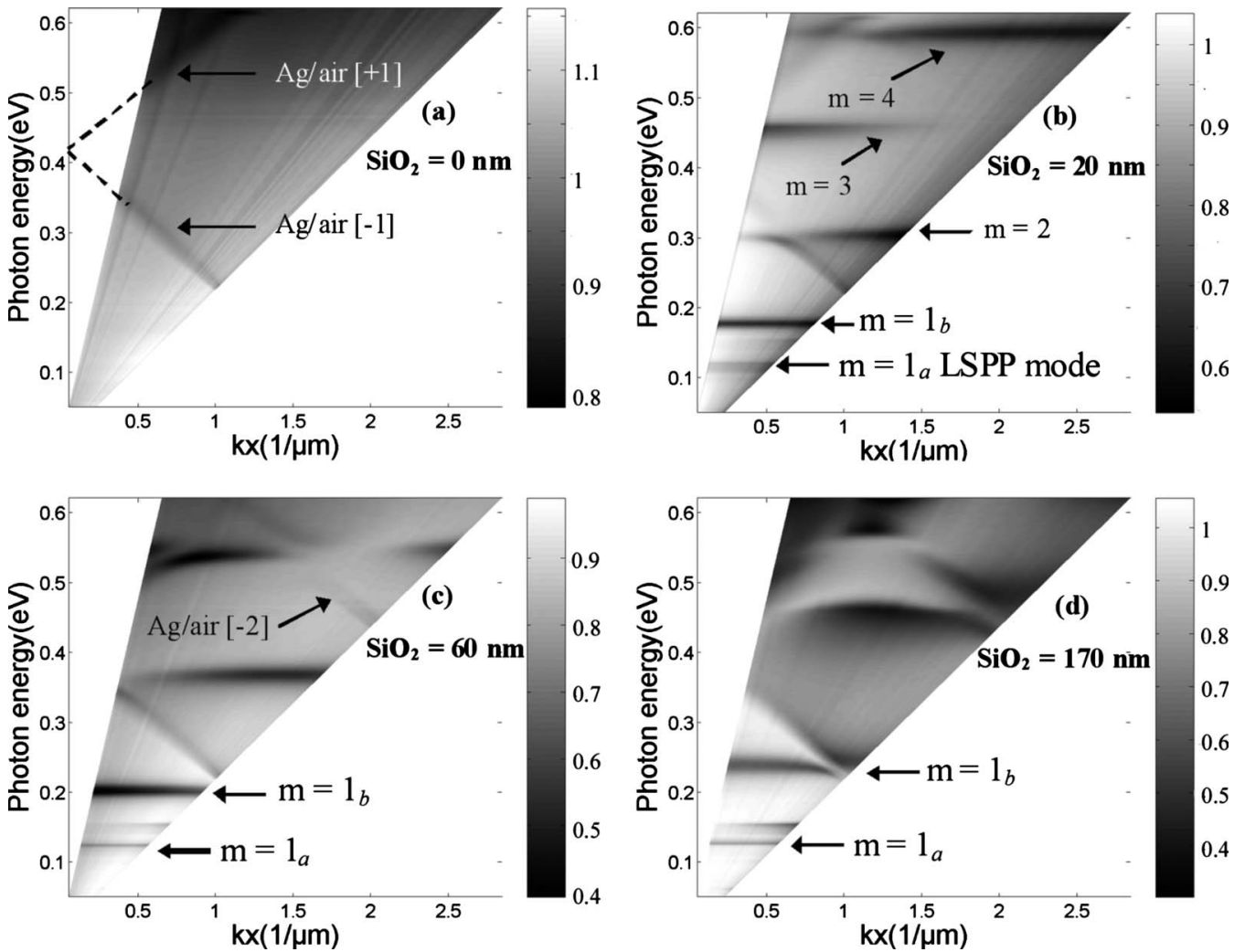

FIG. 2. The dispersion relations of SPs in plasmonic thermal emitters with $\mathrm{SiO}_{2}$ thicknesses of (a) $0 \mathrm{~nm}$, (b) $20 \mathrm{~nm}$, (c) $60 \mathrm{~nm}$, and (c) $170 \mathrm{~nm}$.

$0.42 \mathrm{eV}(\sim 3 \mu \mathrm{m})$, i.e., there exists a reflection dip at $0.42 \mathrm{eV}$ for normal incidence. These two SP modes result from the interaction between incident light and reciprocal lattice vectors, $+G$ and $-G$, at the Ag/air interface. When $t=20 \mathrm{~nm}$, the Ag/air grating-coupled SP modes are still observable, as shown in Fig. 2(b). Furthermore, the dark line around $0.15 \mathrm{eV}$ is attributed to the asymmetric longitudinal optical $\left(\mathrm{AS}_{1} \mathrm{LO}\right)$ phonon vibration mode of the $\mathrm{SiO}_{2}$ layer. ${ }^{13}$ $\mathrm{No} \mathrm{Ag} / \mathrm{SiO}_{2}$ grating-coupled SP mode is observed. However, there exist five additional dark lines at about 0.12, 0.19, $0.31,0.47$, and $0.6 \mathrm{eV}$. These five dark lines are $m=1$, $m=1, m=2, m=3$, and $m=4 \mathrm{Ag} / \mathrm{SiO}_{2} / \mathrm{Ag} \mathrm{LSPP}$ modes, which exhibit the Fabry-Pérot resonance; ${ }^{3,12}$ i.e., the wavelength of incident light $\lambda$ and the $\mathrm{Ag}$ grating line width $d$ satisfy $2 d \approx m \lambda / n_{\text {eff }}$, where $m$ is integer and $n_{\text {eff }}$ is the effective refractive index of $\mathrm{Ag} / \mathrm{SiO}_{2} / \mathrm{Ag} \mathrm{LSPP}$ mode. Two $m=1$ LSPP modes (could be denoted as $m=1_{a}$ and $m=1_{b}$ ) exist at 0.12 and $0.19 \mathrm{eV}$, which is due to the obvious difference in the refraction index $n$ of $\mathrm{SiO}_{2}$ at wavelength of incident light $\lambda=10.65 \mu \mathrm{m}(0.12 \mathrm{eV}, n=2.14)$ and $\lambda=6.5 \mu \mathrm{m}$ $(0.19 \mathrm{eV}, n=1.20) .{ }^{14}$ From Fig. 2(c), as $t$ increases to $60 \mathrm{~nm}$, the dispersion relation of Ag/air grating-coupled SP modes is the same as that in Fig. 2(b) since the Ag/air grating-coupled SP modes are determined by the lattice constant of periodic metallic film. Besides Ag/air [ \pm 1$]$ SP modes, there also exists Ag/air [-2] SP mode. However, the dispersion relations of the LSPP modes all shift to higher energies. When $t$ increases to $170 \mathrm{~nm}, m=1_{a}$ and $m=1_{b}$ LSPP modes shift further to 0.13 and $0.25 \mathrm{eV}$ and other high order LSPP modes shift to higher energies as shown in Fig. 2(d). The shift in the dispersion lines of $\mathrm{Ag} / \mathrm{SiO}_{2} \mathrm{LSPP}$ modes is due to the coupling effect of SPs, which will be discussed later.
Figures 3(a)-3(c) show the thermal radiation spectra of the plasmonic thermal emitters at $260{ }^{\circ} \mathrm{C}$ with $t=20,60$, and $170 \mathrm{~nm}$, respectively. In Fig. 3(c), the emission peaks at 10 and $4.93 \mu \mathrm{m}$ correspond to the $m=1_{a}$ and $m=1_{b}$ $\mathrm{Ag} / \mathrm{SiO}_{2} / \mathrm{Ag} \mathrm{LSPP}$ modes shown in Fig. 2(d). When the emitter is heated, the thermal radiation generated in the $\mathrm{SiO}_{2}$ layer resonates between top and bottom Ag films. Then $\mathrm{Ag} / \mathrm{SiO}_{2} / \mathrm{Ag} \mathrm{LSPPs}$ are induced and converted to light radiation. As $t$ decreases to $20 \mathrm{~nm}$, the emission peaks shift to

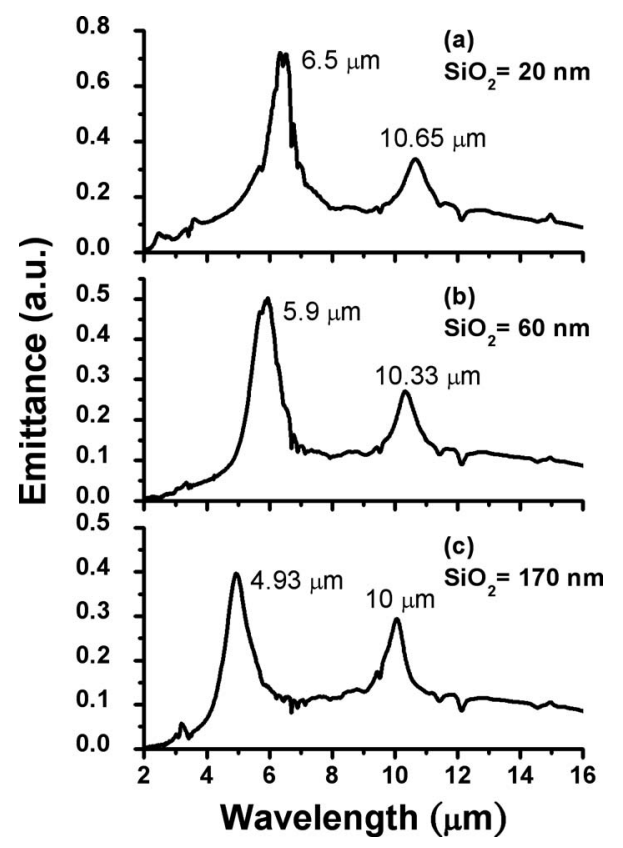

FIG. 3. Measured emission spectra of plasmonic thermal emitters at $260{ }^{\circ} \mathrm{C}$ and the thicknesses of $\mathrm{SiO}_{2}$ are (a) $20 \mathrm{~nm}$, (b) $60 \mathrm{~nm}$, and (c) $170 \mathrm{~nm}$. 

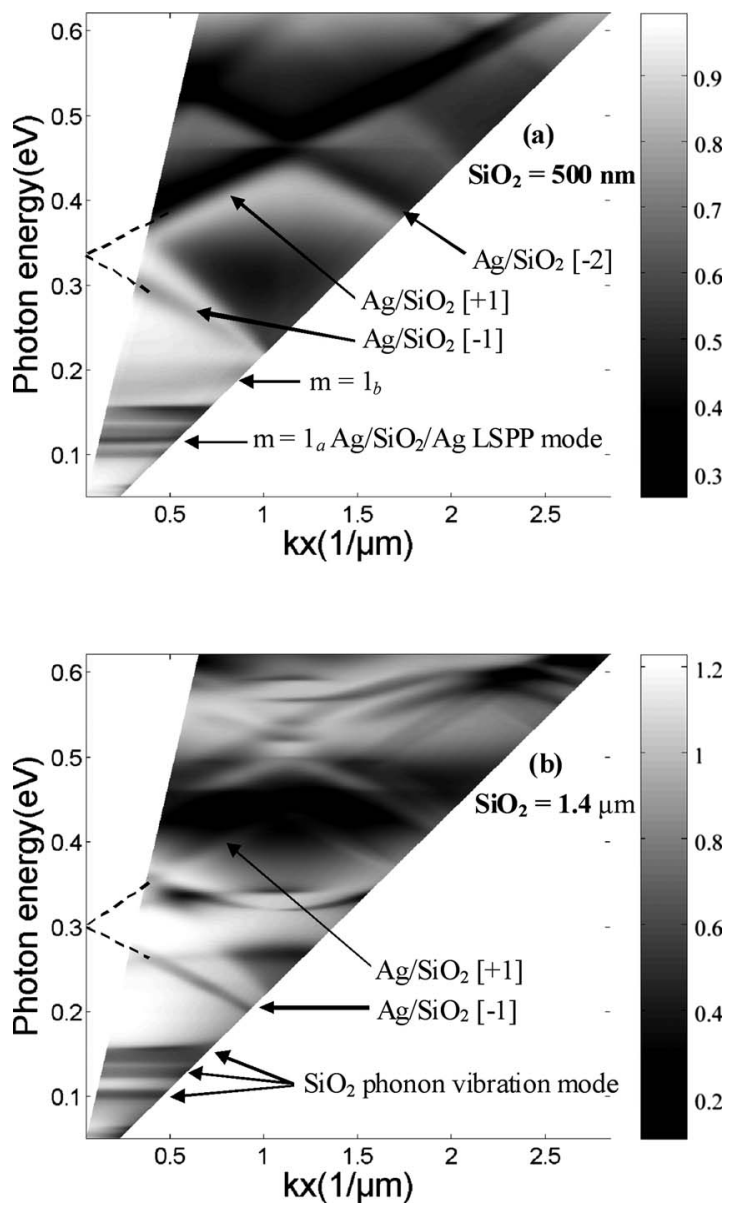

FIG. 4. The dispersion relations of SPs in plasmonic thermal emitters with $\mathrm{SiO}_{2}$ thicknesses of (a) $500 \mathrm{~nm}$ and (b) $1.4 \mu \mathrm{m}$.

10.65 and $6.5 \mu \mathrm{m}$. In our $\mathrm{Ag} / \mathrm{SiO}_{2} / \mathrm{Ag}$ plasmonic thermal emitter, the induced SPs at the top $\mathrm{Ag} / \mathrm{SiO}_{2}$ interface will couple with the SPs at the bottom $\mathrm{Ag} / \mathrm{SiO}_{2}$ interface, ${ }^{15}$ which will increase the effective refraction index. ${ }^{12,16,17}$ The wavelength $\lambda_{p}$ of emission peak resulting from the resonance of LSPPs is expressed as $\lambda_{p} \approx 2 n_{\text {eff }} d$, therefore the redshift in emission peaks represents the increase in $n_{\text {eff }}$, which is consistent with the work demonstrated by Miyazaki et al. ${ }^{12} \mathrm{Ac}-$ cording to the above result, the position of thermal emission peak is not only determined by the Ag grating line width $d$ but also influenced by the thickness of intermediate $\mathrm{SiO}_{2}$ layer.

Figures 4(a) and 4(b) show the dispersion relations of SPs in plasmonic thermal emitters with $t=500 \mathrm{~nm}$ and $1.4 \mu \mathrm{m}$, respectively. The dark lines at about $0.15,0.13$, and $0.10 \mathrm{eV}$ are attributed to the $\mathrm{AS}_{1} \mathrm{LO}$, asymmetric transverse optical, and symmetric stretching phonon vibration modes of the $\mathrm{SiO}_{2}$ layer, respectively. ${ }^{13}$ In Fig. 4(a), $m=1_{a}$ and $m=1_{b}$ $\mathrm{Ag} / \mathrm{SiO}_{2} / \mathrm{Ag}$ LSPP modes become indistinct and $\mathrm{Ag} / \mathrm{SiO}_{2}$ grating-coupled $\mathrm{SP}$ modes $\left(\mathrm{Ag} / \mathrm{SiO}_{2}[ \pm 1]\right.$ and $\mathrm{Ag} / \mathrm{SiO}_{2}$ $[-2])$ appear. The $\mathrm{Ag} / \mathrm{SiO}_{2}[ \pm 1]$ modes intersect with the $y$ axis at about $0.34 \mathrm{eV}$, which is higher than the theoretical value $(0.3 \mathrm{eV})$. In Fig. 4(b), as the thickness of $\mathrm{SiO}_{2}$ in- creased to $1.4 \mu \mathrm{m}$, the $\mathrm{Ag} / \mathrm{SiO}_{2} \mathrm{LSPP}$ modes totally disappear. Moreover, the dispersion relation of $\mathrm{Ag} / \mathrm{SiO}_{2}[ \pm 1]$ modes is almost the same as the theory. When the $\mathrm{SiO}_{2}$ layer is thin, the electromagnetic field exhibits the Fabry-Pérot resonance underneath the $\mathrm{Ag}$ lines due to strong coupling of top and bottom SPs. Therefore $\mathrm{Ag} / \mathrm{SiO}_{2}$ LSPP modes, instead of $\mathrm{Ag} / \mathrm{SiO}_{2}$ grating-coupled $\mathrm{SP}$ modes, dominate at this stage. As the thickness of $\mathrm{SiO}_{2}$ increases, it is found that $\mathrm{Ag} / \mathrm{SiO}_{2} \mathrm{LSPP}$ modes and grating-coupled SP modes exist simultaneously. Furthermore, the interaction between LSPPs and SPs results in the blueshift in $\mathrm{Ag} / \mathrm{SiO}_{2}$ grating-coupled $\mathrm{SP}$ modes. When the thickness of $\mathrm{SiO}_{2}$ is thick enough, coupling of top and bottom SPs can be neglected and $\mathrm{Ag} / \mathrm{SiO}_{2}$ LSPP modes totally disappear, whereas $\mathrm{Ag} / \mathrm{SiO}_{2}$ gratingcoupled SP modes become dominant.

In summary, the dispersion relations and emission spectra of $\mathrm{Ag} / \mathrm{SiO}_{2} / \mathrm{Ag}$ trilayer plasmonic thermal emitters with various $\mathrm{SiO}_{2}$ thicknesses are investigated experimentally. The top and bottom SPs will couple together when $\mathrm{SiO}_{2}$ layer is thin, which increases $n_{\text {eff }}$ and results in the redshift in the thermal emission peak. As the thickness of $\mathrm{SiO}_{2}$ continues to increase, the $\mathrm{Ag} / \mathrm{SiO}_{2} \mathrm{LSPP}$ modes become weaker and finally disappear. When the $\mathrm{SiO}_{2}$ layer is thick enough, the $\mathrm{Ag} / \mathrm{SiO}_{2}$ grating-coupled $\mathrm{SP}$ modes become dominant and the dispersion relation of $\mathrm{Ag} / \mathrm{SiO}_{2}[ \pm 1]$ modes almost coincides with the theory.

The authors would like to thank the National Science Council of the Republic of China for financial support under Contract No. NSC 96-2221-E-002-242.

${ }^{1}$ H. Raether, Surface Plasmons (Springer, Berlin, 1988).

${ }^{2}$ M. Laroche, C. Arnold, F. Marquier, R. Carminati, J.-J. Greffet, S. Collin, N. Bardou, and J.-L. Pelouard, Opt. Lett. 30, 2623 (2005).

${ }^{3}$ C.-M. Wang, Y.-C. Chang, M.-W. Tsai, Y.-H. Ye, C.-Y. Chen, Y.-W. Jiang, Y.-T. Chang, S.-C. Lee, and D. P. Tsai, Opt. Express 15, 14673 (2007).

${ }^{4}$ K. Ikeda, H. T. Miyazaki, T. Kasaya, K. Yamamoto, Y. Inoue, K. Fujimura, T. Kanakugi, M. Okada, K. Hatade, and S. Kitagawa, Appl. Phys. Lett. 92, 021117 (2008).

${ }^{5}$ M. Kreiter, J. Oster, R. Sambles, S. Herminghaus, S. Mittler-Neher, and W. Knoll, Opt. Commun. 168, 117 (1999).

${ }^{6}$ M.-W. Tsai, T.-H. Chuang, C.-Y. Meng, Y.-T. Chang, and S.-C. Lee, Appl. Phys. Lett. 89, 173116 (2006).

${ }^{7}$ M.-W. Tsai, C.-Y. Chen, Y.-W. Jiang, Y.-H. Ye, H.-Y. Chang, T.-H. Chuang, and S.-C. Lee, Appl. Phys. Lett. 91, 213104 (2007).

${ }^{8}$ H. L. Tam, K. F. Li, K. W. Cheah, J. B. Xia, R. Huber, W. H. Wong, and Y. B. Pun, Appl. Phys. Lett. 89, 131123 (2006).

${ }^{9}$ S. A. Kalele, N. R. Tiwari, S. W. Gosavi, and S. K. Kulkarni, J. Nanophotonics 1, 012501 (2007).

${ }^{10}$ R. Biswas, C. G. Ding, I. Puscasu, M. Pralle, M. McNeal, J. Daly, A. Greenwald, and E. Johnson, Phys. Rev. B 74, 045107 (2006).

${ }^{11}$ D. L. C. Chan, M. Soljacic, and J. D. Joannopoulos, Opt. Express 14, 8785 (2006)

${ }^{12}$ H. T. Miyazaki and Y. Kurokawa, Phys. Rev. Lett. 96, 097401 (2006).

${ }^{13}$ C. T. Kirk, Phys. Rev. B 38, 1255 (1988).

${ }^{14}$ D. E. Palik, Handbook of Optical Constants of Solids (Academic, Boston, 1985), pp. 749-763 and 1067.

${ }^{15}$ S. A. Darmanyan and A. V. Zayats, Phys. Rev. B 67, 035424 (2003).

${ }^{16}$ S. Collin, F. Pardo, and J.-L. Pelouard, Opt. Express 15, 4310 (2007).

${ }^{17}$ C.-Y. Chen, M.-W. Tsai, Y.-W. Jiang, Y.-H. Ye, Y.-T. Chang, and S.-C. Lee, Appl. Phys. Lett. 91, 243111 (2007). 